\section{Insomnia and Cardiac Events in Patients With Heart Failure}

\section{To the Editor:}

Kanno et al conducted a prospective study on the association between insomnia and subsequent cardiac events, including death, among 1,011 patients with heart failure (HF). ${ }^{1}$ The authors followed for more than 2 years on average, and the adjusted hazard ratio (HR) (95\% confidence interval $[\mathrm{CI}]$ ) of insomnia for cardiac events, including cardiac death and/or worsening HF, was 1.899 (1.333$2.705)$ by Cox regression analysis. I have some concerns about their study.

First, the authors identified 318 cardiac events as cardiac death $(n=151)$ or worsening HF $(n=236)$. Cause of HF would vary according to the severity of the underlying disease, and thus the 2 events should be independently evaluated in relation to insomnia.

Second, the authors described in their study's limitations that psychiatric parameters such as depression and cognitive impairment were not included as confounders for insomnia. These variables would fluctuate in the course of follow-up and appropriate adjustment would be required to assess the association between insomnia and cardiac events in patients with HF.

Third, Clark et al examined the effect of impaired sleep in patients with acute myocardial infarction (AMI). ${ }^{2}$ The HR $(95 \% \mathrm{CI})$ of disturbed sleep for non-fatal HF was 2.43
(1.18-4.97) in women. In contrast, a significant increase in mortality rate was observed with regard to impaired awakening in men. The sex difference was not recognized in study by Kanno et al. On this point, Condén and Rosenblad conducted a prospective study and their HR (95\% CI) of male against female among patients with AMI for allcause death was $3.359(2.272-4.967) .{ }^{3}$ I speculate that the sex difference should be specified once again by stratification of cardiac events as a dependent variable. At that time, smoking and drinking habits should be considered as independent variables.

\section{Disclosure Statement}

The author has indicated no financial support.

\section{Conflicts of Interest}

There is no conflict of interest in this study.

\section{References}

1. Kanno Y, Yoshihisa A, Watanabe S, Takiguchi M, Yokokawa T, Sato A, et al. Prognostic significance of insomnia in heart failure. Circ J 2016; 80: 1571-1577.

2. Clark A, Lange T, Hallqvist J, Jennum P, Rod NH. Sleep impairment and prognosis of acute myocardial infarction: A prospective cohort study. Sleep 2014; 37: 851-858.

3. Condén E, Rosenblad A. Insomnia predicts long-term all-cause mortality after acute myocardial infarction: A prospective cohort study. Int J Cardiol 2016; 215: 217-222.

Tomoyuki Kawada, MD

Department of Hygiene and Public Health, Nippon Medical School, Tokyo, Japan

Received November 14, 2016; accepted November 17, 2016; released online December 14, 2016

Mailing address: Tomoyuki Kawada, MD, Department of Hygiene and Public Health, Nippon Medical School, 1-1-5 Sendagi, Bunkyo-ku, Tokyo 113-8602, Japan. E-mail: kawada@nms.ac.jp

ISSN-1346-9843 All rights are reserved to the Japanese Circulation Society. For permissions, please e-mail: cj@j-circ.or.jp 